Gut and Liver, Vol. 9, No. 6, November 2015, pp. 791-799

\title{
A Comparison of Preoperative Biliary Drainage Methods for Perihilar Cholangiocarcinoma: Endoscopic versus Percutaneous Transhepatic Biliary Drainage
}

\author{
Kwang Min Kim*, Ji Won Park ${ }^{\dagger}$, Jong Kyun Lee ${ }^{\dagger}$, Kwang Hyuck Lee ${ }^{\dagger}$, Kyu Taek Lee ${ }^{\dagger}$, and Sang Goon Shim* \\ ${ }^{*}$ Department of Medicine, Samsung Changwon Hospital, Sungkyunkwan University School of Medicine, Changwon, and ${ }^{\dagger}$ Department of \\ Medicine, Samsung Medical Center, Sungkyunkwan University School of Medicine, Seoul, Korea
}

\begin{abstract}
Background/Aims: Controversy remains over the optimal approach to preoperative biliary drainage in patients with resectable perihilar cholangiocarcinoma. We compared the clinical outcomes of endoscopic biliary drainage (EBD) with those of percutaneous transhepatic biliary drainage (PTBD) in patients undergoing preoperative biliary drainage for perihilar cholangiocarcinoma. Methods: A total of 106 consecutive patients who underwent biliary drainage before surgical treatment were divided into two groups: the PTBD group $(n=62)$ and the EBD group $(n=44)$. Results: Successful drainage on the first attempt was achieved in 36 of 62 patients (58.1\%) with PTBD, and in 25 of 44 patients (56.8\%) with EBD. There were no significant differences in predrainage patient demographics and decompression periods between the two groups. Procedure-related complications, especially cholangitis and pancreatitis, were significantly more frequent in the EBD group than the PTBD group (PTBD vs EBD: $22.6 \%$ vs $54.5 \%, p<0.001)$. Two patients $(3.8 \%)$ in the PTBD group experienced catheter tract implantation metastasis after curative resection during the follow-up period. Conclusions: EBD was associated with a higher risk of procedure-related complications than PTBD. These complications were managed properly without severe morbidity; however, in the PTBD group, there were two cases of cancer dissemination along the catheter tract. (Gut Liver 2015;9:791-799)
\end{abstract}

Key Words: Complications; Endoscopic biliary drainage; Perihilar cholangiocarcinoma; Percutaneous transhepatic biliary drainage; Preoperative biliary drainage

\section{INTRODUCTION}

Although there is debate about the effect of preoperative biliary drainage on surgical outcome in patients with malignant biliary obstruction, it has been demonstrated that liver dysfunction caused by obstructive jaundice can be a significant risk factor in major liver resection. ${ }^{1-3}$ Especially in the case of perihilar cholangiocarcinoma, for which extended hepatectomy may be required to provide the best chance for cure, it is preferable to perform preoperative drainage of the future remnant liver to preserve postoperative liver function. Furthermore, selective cholangiography via a preoperative biliary drainage catheter often provides more precise information about the extent of the tumor along the bile duct. ${ }^{4}$

However, controversy remains over how best to perform preoperative biliary drainage in patients with perihilar cholangiocarcinoma. There have been several disputes over the clinical advantages of percutaneous transhepatic biliary drainage (PTBD) versus endoscopic biliary drainage (EBD), the latter which can be achieved by either endoscopic retrograde biliary drainage (ERBD) or endoscopic nasobiliary drainage (ENBD). PTBD has been the preferred method for initial preoperative biliary drainage. ${ }^{5-7}$ However, it can be complicated by tube dislodgement, vascular injury, and portal vein thrombosis. ${ }^{8-10}$ It has been also suggested that catheter tract implantation metastasis can occur after the PTBD procedure. ${ }^{11,12}$ Because EBD is relatively less invasive and avoids these drawbacks of PTBD, it is increasingly being attempted in the clinical setting. Still, some disadvantages must be considered. Because endoscopic retrograde cholangiopancreatography (ERCP) is basically a prerequisite for performing EBD, ERCP-related risks such as duodenal perforation,

\footnotetext{
Correspondence to: Jong Kyun Lee

Department of Medicine, Samsung Medical Center, Sungkyunkwan University School of Medicine, 81 Irwon-ro, Gangnam-gu, Seoul 06351, Korea

Tel: +82-2-3410-3409, Fax: +82-2-3410-6983, E-mail: jongk.lee@samsung.com

Received on July 3, 2014. Revised on November 29, 2014. Accepted on December 16, 2014. Published online June 19, 2015

pISSN 1976-2283 eISSN 2005-1212 http://dx.doi.org/10.5009/gnl14243

Kwang Min Kim and Ji Won Park contributed equally to this study as first authors.

() This is an Open Access article distributed under the terms of the Creative Commons Attribution Non-Commercial License (http://creativecommons.org/licenses/by-nc/4.0) which permits unrestricted non-commercial use, distribution, and reproduction in any medium, provided the original work is properly cited.
} 
cholangitis, and acute pancreatitis should be taken into consideration when performing EBD. Additionally, failure of cannulation disallows subsequent endoscopic intervention. Despite controversy concerning these two main approaches, few studies have compared the clinical outcomes between EBD and PTBD, and no consensus has been reached regarding which method should be selected for preoperative biliary drainage for resectable perihilar cholangiocarcinoma. Our purpose in this study was therefore to compare the effectiveness and safety of EBD with that of PTBD in patients undergoing preoperative biliary drainage for perihilar cholangiocarcinoma.

\section{MATERIALS AND METHODS}

\section{Patients}

A total of 106 consecutive patients who underwent biliary drainage before surgical treatment for perihilar cholangiocarcinoma at Samsung Medical Center, Seoul, Korea, between January 2000 and April 2012, were retrospectively reviewed. These patients were classified into two groups: a PTBD group consisting of 62 patients who underwent PTBD as the initial preoperative procedure, and an EBD group comprising 44 patients who underwent EBD as the initial preoperative procedure. Among the 44 patients in the EBD group, ERBD was carried out in nine patients, whereas the remaining 35 patients underwent ENBD. Combinations of computed tomography (CT), magnetic resonance imaging (MRI), endoscopic ultrasonography, and cholangiography were used as diagnostic aids to determine resectability of perihilar cholangiocarcinoma before surgery. Ethics Committee of Samsung Medical Center approved this study protocol and the study was conducted in accordance with the principles of the Declaration of Helsinki.

\section{PTBD and EBD procedures}

Representative cases that underwent PTBD and EBD procedures are shown in Fig. 1. PTBD was always performed on patients under local anesthesia with application of meperidine (25 to $50 \mathrm{mg}$ ) by several interventional radiologists with more than 10 years of experience. After draping the operation field, biliary duct was punctured using a 21-gauge Chiba needle through a right or left intercostal percutaneous approach under ultrasound and fluoroscopic guidance. After puncturing the target duct and confirming bile juice flow from the Chiba needle, cholangiography was performed to localize the site of obstruction by injecting contrast material gently, under fluoroscopic guidance. Then, an 8.5-F drainage catheter with multiple side holes (Cook Medical Inc., Bloomington, IN, USA) was placed and was not exchanged before surgery. EBD was performed using a duodenoscope with a 4.2-mm operative channel (TFJ 240 or TJF 260; Olympus, Tokyo, Japan) by three experienced endoscopists (K.H.L., K.T.L., and J.K.L.). All patients were sedated using midazolam (2 to $5 \mathrm{mg}$ ) with meperidine ( 25 to $50 \mathrm{mg}$ ) administered intravenously and appropriate cardiopulmonary monitoring. A small endoscopic sphinctertomy was performed in all patients to facilitate introduction of the various catheters and prevent pancreatitis. After successful biliary cannulation, a guidewire was inserted into the future remnant lobe and then a selective cholangiogram was taken to localize the site of obstruction. Distal end of the ERBD (7 or $8.5 \mathrm{~F}$ ) or ENBD (5 or $7 \mathrm{~F}$ ) catheter was advanced through the guidewire into the left or right-side hepatic duct and upward to the biliary stricture, preferentially to the future remnant lobe.

\section{Data collection and assessment of outcomes}

Preoperative data were collected from laboratory, radiologic, endoscopic, and surgical databases. Demographics and comorbidity were analyzed. All procedure-related complications including cholangitis, pancreatitis, PTBD or EBD tube dislocation/removal, tube occlusion, nerve injury and bleeding, and perforation were also recorded. Procedure-related cholangitis and pancreatitis were assessed based on consensus criteria. ${ }^{13}$ Procedure-related bleeding after biliary drainage was defined as bleeding that required transfusion or additional intervention, or as bleeding that caused hemoglobin levels to fall by $2 \mathrm{~g} / \mathrm{dL}$.

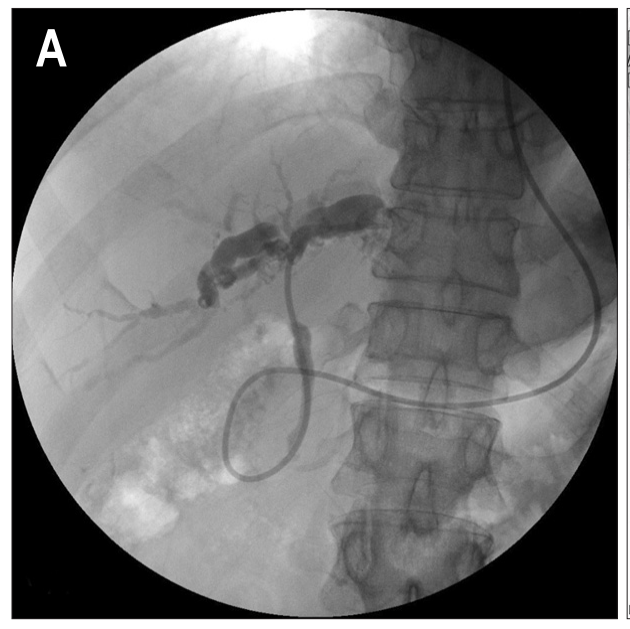

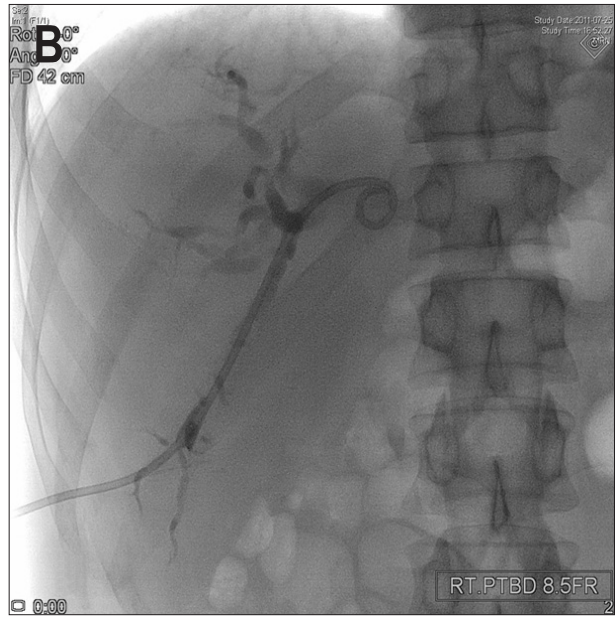

Fig. 1. Two representative cases of preoperative biliary drainage for perihilar cholangiocarcinoma. Cholangiogram obtained using a 7-F endoscopic nasobiliary drainage tube that was advanced through the malignant stricture into the left intrahepatic duct of the future remnant liver (A). Fluoroscopic image showing the placement of the 8.5F percutaneous transhepatic biliary drainage tube, which was deployed into the right intrahepatic duct (B). 
Period of decompression was defined as either the number of days from initial biliary drainage until the date of serum total bilirubin reduction to $1.5 \mathrm{mg} / \mathrm{dL}$ or lower, or the number of days from initial biliary drainage until date of operation. All references to bilirubin values refer to serum total bilirubin. We have defined drainage type conversion as to PTBD or EBD from EBD or PTBD, respectively, for rescue therapy when successful drainage was not achieved using the initial method. Total operation time was recorded as the time from skin incision to skin closure. Postoperative complications including infection, liver failure, hemorrhage, and bile leakage were graded according to the established Clavien-Dindo classification; grade 3 and greater complications were regarded as surgical complications leading to severe morbidity. ${ }^{14}$ We used consensus definitions and severity gradings of posthepatectomy liver failure outlined by the International Study Group of Liver Surgery. ${ }^{15}$

\section{Follow-up and recurrence}

All patients who underwent surgery for perihilar cholangiocarcinoma underwent routine CT or MRI and a checkup for biochemical parameters at an outpatient clinic, every 6 months for the first 2 years and annually thereafter to check for local recurrence, lymph node involvement, and distant metastasis. Additional information regarding current status or death of the patients was obtained by direct contact with the referring physician or by telephone.

\section{Statistical analysis}

Statistical analyses were performed using PASW Statistics
17.0 (SPSS Inc., Chicago, IL, USA). Chi-square or Fisher exact tests were used for categorical variables, and Student t-tests or Mann-Whitney U tests were used for continuous variables. $\mathrm{Cu}$ mulative patient survival was compared using the Kaplan-Meier method and the log-rank test. p-values $<0.05$ were considered statistically significant.

\section{RESULTS}

\section{Patient characteristics}

Baseline demographic and clinical characteristics of all 106 patients in this study who received preoperative biliary drainage are shown in Table 1. Perihilar cholangiocarcinomas were classified according to the Bismuth-Corlette classification system: approximately 80\% were either type III (n=67/106, 63.2\%) or type IV ( $n=21 / 106,19.8 \%)$, followed by type II ( $n=16 / 106$, 15.1\%). The combined total of type III and IV cases, as a ratio to the total population, did not differ significantly between the two groups (PTBD vs EBD: 83.9\% (52/62) vs 81.8\% (36/44), $\mathrm{p}=0.798)$. Thirty-seven of 62 patients $(59.7 \%)$ in the PTBD group and 24 of 44 patients (54.5\%) in the EBD group underwent preoperative biliary drainage at a tertiary referral center. There was no significant difference between the two groups in the type of hospital where the initial drainage procedure was undertaken. Preoperative portal vein embolization was performed in $21.0 \%$ $(13 / 62)$ of PTBD cases and $18.2 \%(8 / 44)$ of EBD cases to improve the function of the future remnant liver. No significant difference was observed in peak serum bilirubin level before drainage between the two groups (PTBD vs EBD: $13.7 \pm 6.7 \mathrm{mg} /$

Table 1. Baseline Characteristics of Patients Who Underwent Preoperative Biliary Drainage

\begin{tabular}{|c|c|c|c|}
\hline Variable & PTBD $(n=62)$ & $\operatorname{EBD}(n=44)$ & p-value \\
\hline Age, yr & $62(46-89)$ & $63(42-79)$ & 0.802 \\
\hline Sex, male & $38(61.3)$ & $30(68.2)$ & 0.466 \\
\hline Bismuth-Corlette classification & $0 / 10 / 38 / 14$ & $2 / 6 / 29 / 7$ & $0.782^{*}$ \\
\hline I & 0 & $2(4.6)$ & \\
\hline II & $10(16.1)$ & $6(13.6)$ & \\
\hline III & $38(61.3)$ & 29 (65.9) & \\
\hline IV & $14(22.6)$ & $7(15.9)$ & \\
\hline Peak serum bilirubin before drainage, $\mathrm{mg} / \mathrm{dL}$ & $13.7 \pm 6.7$ & $10.3 \pm 7.1$ & 0.061 \\
\hline \multicolumn{4}{|l|}{ Comorbidity } \\
\hline Hypertension & $25(40.3)$ & $9(20.5)$ & 0.031 \\
\hline Diabetes mellitus & $16(25.8)$ & $8(18.2)$ & 0.355 \\
\hline Liver cirrhosis & 0 & $1(2.3)$ & 0.415 \\
\hline Chronic kidney disease & 0 & $1(2.3)$ & 0.415 \\
\hline Cardiovascular disease & $3(4.8)$ & 0 & 0.265 \\
\hline Initial drainage at tertiary facility & $37(59.7)$ & $24(54.5)$ & 0.598 \\
\hline Preoperative PVE & 13 (21.0) & $8(18.2)$ & 0.723 \\
\hline
\end{tabular}

Age is presented as the median (range); other variables are presented as number (\%) or mean \pm standard deviation. PTBD, percutaneous transhepatic biliary drainage; EBD, endoscopic biliary drainage; PVE, portal vein embolization. *p-value: I, II vs III, IV. 
$\mathrm{dL}$ vs $10.3 \pm 7.1 \mathrm{mg} / \mathrm{dL}, \mathrm{p}=0.061$ ). Forty-four patients had single or multiple medical comorbidities including hypertension, diabetes mellitus, liver cirrhosis, chronic kidney disease, or cardiovascular disease. Hypertension was significantly more common in the PTBD group than in the EBD group (40.3\% vs $20.5 \%$, $\mathrm{p}=0.031$ ). No other underlying diseases showed a significant difference between these two groups.

\section{Technical and therapeutic outcomes}

Technical outcomes and complications according to type of preoperative biliary drainage are shown in Table 2. 62.9\% (39/62) of PTBD patients and 59.1\% (26/18) of EBD patients underwent unilateral biliary drainage. There was no significant difference between the two groups regarding whether bilateral or unilateral drainage of the hepatic lobes was employed. Serum bilirubin level decreased significantly after preoperative biliary drainage in both groups (from $13.7 \pm 6.7$ to $3.08 \pm 2.0 \mathrm{mg} / \mathrm{dL}$ in the PTBD group, $\mathrm{p}<0.001$, and from $10.3 \pm 7.1$ to $2.36 \pm 1.4 \mathrm{mg} / \mathrm{dL}$ in the EBD group, $\mathrm{p}<0.001)$. Although the preoperative serum bilirubin level of the PTBD group was significantly higher than that of the EBD group (PTBD vs EBD: $3.08 \pm 2.0 \mathrm{mg} / \mathrm{dL}$ vs $2.36 \pm 1.4$ $\mathrm{mg} / \mathrm{dL}, \mathrm{p}=0.044$ ), differences in serum bilirubin level before and after biliary drainage were not significant (PTBD vs EBD: $10.40 \pm 6.3 \mathrm{mg} / \mathrm{dL}$ vs $7.9 \pm 6.8 \mathrm{mg} / \mathrm{dL}, \mathrm{p}=0.145)$. Successful drainage on first attempt was achieved in 36 of 62 patients (58.1\%) who underwent PTBD. This did not differ significantly from the successful proportion of first attempts in patients who under- went $\operatorname{EBD}$ (25/44, 56.8\%). In contrast, 41.9\% of patients who underwent PTBD and $43.2 \%$ of patients who underwent EBD required further intervention. Rate of drainage type conversion in the EBD group was $38.6 \%$, which was significantly higher than that in the PTBD group (EBD vs PTBD: 38.6\% [17/44] vs 6.5\% [4/62], $\mathrm{p}<0.001)$. Median period of biliary decompression in patients who underwent PTBD was 20 days (range, 3 to 56 days) compared to 18 days (range, 2 to 68 days) for patients who underwent EBD. This difference was not significant $(p=0.113)$.

\section{Complications}

Procedure-related complications occurred in 14 of 62 patients $(22.6 \%)$ in the PTBD group and 24 of 44 patients (54.5\%) in the EBD group, thus complications were significantly more frequent in the EBD group (PTBD vs EBD: 22.6\% vs 54.5\%, p<0.001). The most common complication in the PTBD group was tube dislocation (14.5\%), followed by cholangitis (8.1\%) and tube occlusion (3.2\%). In contrast, in the EBD group, cholangitis was most common at a frequency of $38.6 \%$, followed by pancreatitis with a frequency of $20.5 \%$. These rates were significantly higher than those observed in the PTBD group $(\mathrm{p}<0.001$ and $\mathrm{p}<0.001$ for cholangitis and pancreatitis, respectively). In the EBD group, all patients who developed acute pancreatitis were treated successfully by conservative methods with no additional intervention, whereas two of 16 patients (12.5\%) who developed cholangitis required reintervention. Three patients who developed postERCP hemorrhage in the EBD group recovered uneventfully

Table 2. Comparison of the Technical and Clinical Outcomes according to the Type of Preoperative Biliary Drainage

\begin{tabular}{lccc}
\hline \multicolumn{1}{c}{ Variable } & PTBD (n=62) & EBD (n=44) & p-value \\
\hline Initial technical success & $36(58.1)$ & $25(56.8)$ & 0.898 \\
Total no. of procedures, mean & 1.6 & 1.6 & 0.805 \\
Period of biliary decompression, days & $20(3-56)$ & $18(2-68)$ & 0.113 \\
Postdrainage laboratory findings & & & 0.044 \\
Serum total bilirubin, mg/dL & $3.08 \pm 2.0$ & $2.36 \pm 1.4$ & 0.878 \\
ALP, IU/L & $230 \pm 123$ & $224 \pm 119$ & 0.492 \\
AST, IU/L & $52.8 \pm 31.0$ & $48.7 \pm 28.6$ & 0.090 \\
ICG at 15 min, \% & $21.9 \pm 19.2$ & $16.0 \pm 11.4$ & 0.001 \\
No. of patients with complications & $14(22.6)$ & $24(54.5)$ & $<0.001$ \\
Cholangitis & $5(8.1)$ & $16(38.6)$ & $9(20.5)$ \\
Pancreatitis & 0 & $3(6.8)$ & $<0.001$ \\
Tube dislocation/removal & $9(14.5)$ & $1(2.3)$ & 0.352 \\
Tube occlusion & $2(3.2)$ & 0 & 1.000 \\
Nerve injury & $1(1.6)$ & $3(6.8)$ & 1.000 \\
Bleeding & $1(1.6)$ & $26(21 / 5) / 18$ & 0.305 \\
Drained area, unilateral (remnant/resected)/bilateral & $39(31 / 8) / 23$ & $17(38.6)$ & $0.820^{*}$ \\
Drainage type conversion & $4(6.5)$ & $<0.001$ \\
\hline
\end{tabular}

The period of biliary decompression is presented as the median (range); other variables are presented as number (\%) or mean \pm SD. PTBD, percutaneous transhepatic biliary drainage; EBD, endoscopic biliary drainage; ALP, alkaline phosphatase; AST, aspartate transaminase; ICG, indocyanine green. *p-value: unilateral vs bilateral. 
without transfusion. None of the patients in the EBD group experienced major complications such as significant bleeding requiring transfusion, bowel perforation, or bile peritonitis. One patient (1.6\%) in the PTBD group developed hemobilia following PTBD and required transfusion, but this resolved after angiographic embolization of the hepatic artery pseudoaneurysm. Two patients had a reduction in biliary drainage volume 3 days following PTBD, but drainage volume returned to normal following revision of the PTBD tube and biliary flushing. One patient (1.6\%) who underwent PTBD suffered from cutaneous paresthesia on the skin puncture site, which recovered spontaneously. No procedure-related mortalities occurred in either group.

\section{Operative outcomes}

Operative outcomes, including postoperative morbidity and mortality in each group, are presented in Table 3. Most patients in both groups underwent extended hepatic resection including extended right hepatectomies (Segments IV-VIII \pm I) and extended left hepatectomies (Segments II-V, VIII \pm I) with curative intent combined with regional lymph node dissection (PTBD vs ENBD: 53 (85.5\%) vs 42 (95.5\%), p=0.117). Nine (8.5\%) and two (1.9\%) patients underwent right and left hemihepatectomy, respectively. Curative (R0) resection was achieved in 92 patients (86.8\%). Postoperative complications occurred in 16 of 62 patients (25.8\%) in the PTBD group and 16 of 44 (36.4\%) in the
EBD group. This difference was not significant ( $\mathrm{p}=0.243)$. The most common postoperative complication was intra-abdominal abscess formation $(n=18,17 \%)$, followed by posthepatectomy liver failure at grade B and C severity ( $\mathrm{n}=14,13.2 \%)$. There were $10(16.1 \%)$ severe morbidity cases (grade 3 or higher surgical complications) in the PTBD group and nine (20.5\%) in the EBD group; this difference was not statistically significant $(p=0.502)$. Average duration of hospital stay after surgery was not significantly different between the two groups at $18.3 \pm 16.3$ days for the PTBD group and $18.5 \pm 10.7$ days for the EBD group ( $p=0.502$ ). There were five cases of mortality in the perioperative period. Four patients developed posthepatectomy liver failure and died of multiorgan failure, and one patient died of postoperative sepsis following acute respiratory failure. There was no significant difference in postoperative mortality between the two groups (PTBD vs EBD: three (4.8\%) vs two (4.5\%) cases, $\mathrm{p}=1.000)$.

\section{Follow-up and tumor recurrence}

Median follow-up time for all patients was 25.8 months (range, 1 to 86 months). We identified 92 patients who underwent curative (R0) resection for perihilar cholangiocarcinoma. Of these patients, 58 (63.0\%) developed tumor recurrence after curative resection. Among those patients who experienced recurrence, the median time to recurrence was 15.1 months; $37.9 \%$ of recurrences occurred within 12 months, and the latest recurrence was observed at 77 months. Cumulative recurrence rate in the

Table 3. Comparison of the Operative Outcomes and Tumor Recurrence Rates according to the Type of Preoperative Biliary Drainage

\begin{tabular}{lccc}
\hline \multicolumn{1}{c}{ Variable } & PTBD (n=62) & EBD (n=44) & p-value \\
\hline Surgical procedure & & & \\
$\quad$ Extended hepatectomy* & $53(85.5)$ & $2(95.5)$ & 0.117 \\
$\quad$ Right hepatectomy & $7(11.3)$ & 0 & 0.300 \\
$\quad$ Left hepatectomy & $2(3.2)$ & $398.6 \pm 85.7$ & 0.510 \\
Total operation time, min & $375.2 \pm 76.6$ & $16(36.4)$ & 0.144 \\
No. of patients with postoperative complications & $16(25.8)$ & $11(25.0)$ & 0.243 \\
$\quad$ Intra-abdominal abscess formation & $7(11.3)$ & $6(13.6)$ & 0.073 \\
$\quad$ Liver failure & $8(12.9)$ & $1(2.3)$ & 1.000 \\
$\quad$ Wound infection & $3(4.8)$ & 0 & 0.640 \\
$\quad$ Bile leakage & $4(6.5)$ & $18.5 \pm 10.7$ & 0.140 \\
Postoperative hospital stay, days & $18.3 \pm 16.3$ & $9(20.5)$ & 0.502 \\
Severe morbidity & $10(16.1)$ & $2(4.5)$ & 0.567 \\
Postoperative mortality & $3(4.8)$ & $40(90.9)$ & 1.000 \\
Curative (Ro) resection & $52(83.9)$ & $28(70.0)$ & 0.292 \\
Tumor recurrence after curative resection & $30(57.7)$ & $2(3.2)$ & $29(65.9)$ \\
Catheter tract metastasis & $38(61.3)$ & $21(1-86)$ & 0.278 \\
Overall tumor recurrence & $19(1-82)$ & & 0.510 \\
Survival, median (range), mo & & $0.951^{\dagger}$ & 0.959 \\
\hline All & &
\end{tabular}

All of the results are presented as number (\%) or mean \pm SD.

${ }^{*}$ Extended hepatectomy: extended right hepatectomies (Segments IV-VIII $\pm \mathrm{I}$ ) or extended left hepatectomies (Segments II-V, VIII $\pm \mathrm{I}$ ); ${ }^{\dagger} \mathrm{p}$-value: cumulative tumor recurrence rates were compared using the Kaplan-Meier method. 

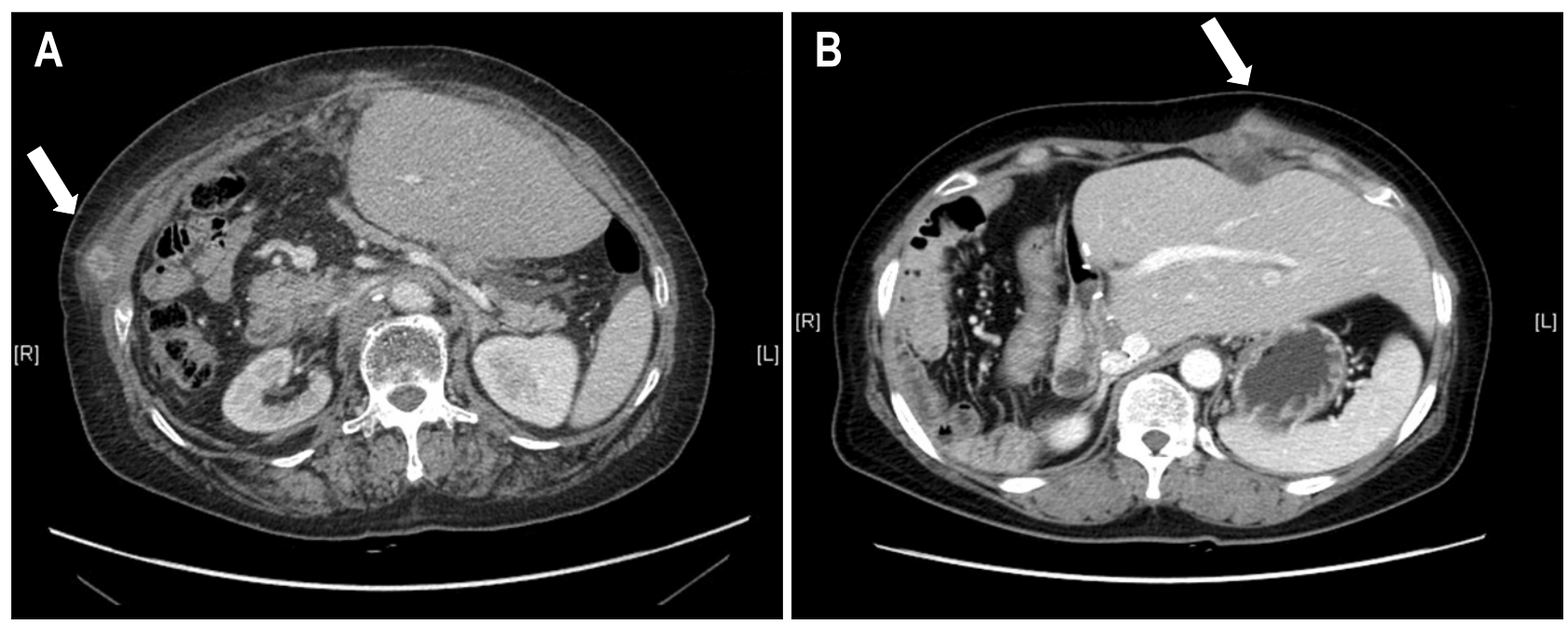

Fig. 2. Two cases of catheter tract implantation metastasis (white arrow) after percutaneous transhepatic biliary drainage, which was detected on follow-up computed tomography scans. Extended right hepatectomy was performed with curative intent for Bismuth-Corlette type IIIa perihilar cholangiocarcinoma in both cases, and catheter tract metastases were found 5 and 16 months after surgery, respectively (A, B).

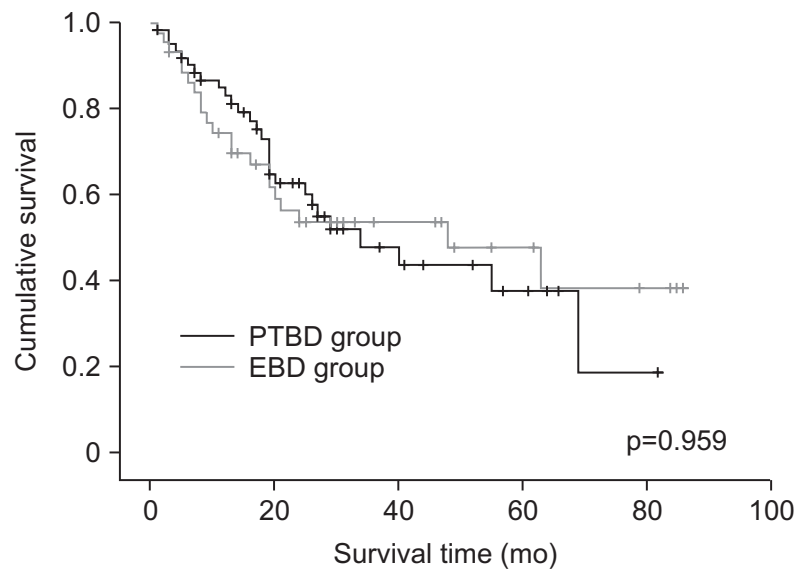

Fig. 3. Kaplan-Meier survival curves of the cumulative survival of patients who underwent preoperative biliary drainage. No significant difference was observed between the percutaneous transhepatic biliary drainage (PTBD) group and the endoscopic biliary drainage (EBD) group.

PTBD group was 57.7\%, which was not significantly different from $70.0 \%$ in the EBD group ( $\mathrm{p}=0.278$ ). Moreover, of 52 patients who underwent curative resection in the PTBD group, two (3.8\%) developed catheter tract implantation metastasis during the follow-up period. There was no evidence of synchronous PTBD tract recurrence at the time of primary tumor resection in these two patients; however, they showed catheter tract metastasis at 5 and 16 months after surgery, respectively (Fig. 2). The mean time to recurrence was shorter in these two patients than in the other 56 patients who experienced tumor recurrence without catheter tract metastasis (10.5 months vs 15.3 months). However, this difference was not statistically significant $(\mathrm{p}=0.848)$. We performed a multivariate analysis of potential risk factors related to catheter tract implantation metastasis, but identified no significant factor associated with the presence of catheter tract metastasis. With regard to mortality, 49 of 106 patients (46.2\%) who underwent preoperative biliary drainage died of the disease during follow-up. Of these 49 patients, 35 experienced recurrence after curative resection. Median survival time was 19 months (range, 1 to 82 months) in the PTBD group and 21 months (range, 1 to 86 months) in the EBD group. Kaplan-Meier survival curves were constructed to analyze survival in the two groups (Fig. 3). Survival did not differ significantly according to type of preoperative biliary drainage $(p=0.959)$.

\section{DISCUSSION}

It is widely accepted that extended liver resection in the treatment of perihilar cholangiocarcinoma may increase the rate of negative histologic margins and improve survival. ${ }^{16-18}$ However, more aggressive hepatic resection in this setting is also associated with high postoperative morbidity and mortality. ${ }^{19,20}$ For this reason, several attempts have been made to improve perioperative outcomes.

To date, preoperative biliary drainage and ipsilateral portal vein embolization have been proposed as methods to improve the function of the future remnant liver. The potential advantages of preoperative biliary drainage include its potential to reverse cholestasis-associated hepatic and synthetic toxicity as well as improve nutritional status and immune function. ${ }^{21,22}$ In the present study, most patients (89.6\%, 95/106) underwent major hepatectomy with curative intent and of those, 22.1\% (21/95) received embolization of the hemi-liver to be resected. None of the patients who underwent ipsilateral portal vein embolization prior to resection showed perioperative mortality, which is consistent with an earlier report that reported that the combined strategy of preoperative biliary drainage and portal vein em- 
bolization can reduce mortality in patients undergoing major hepatectomy. ${ }^{23,24}$

While PTBD is conventionally used as a preoperative drainage method for perihilar cholangiocarcinoma, some studies have demonstrated the usefulness of EBD ${ }^{6,8}$ In addition, it was suggested that unilateral EBD decompresses the future remnant liver effectively and thus promotes its increased use..$^{25}$ However, EBD is often laborious and associated with insufficient drainage in cases with complex biliary obstruction patterns, so PTBD is still preferably performed in patients with perihilar cholangiocarcinoma. PTBD provides extensive drainage of the liver via multiple catheters and allows more precise anatomic delineation of the tumor by contrast injection, and may offer an additional exploratory advantage during surgical observation of the hilar area of the liver. ${ }^{6,26}$ It has also been found that the reintervention rate, whether to the same or an alternate drainage type, is generally lower in PTBD than in EBD. ${ }^{8,26}$ We found a lower conversion rate in the PTBD group than the EBD group, consistent with these previous studies. Conversion to PTBD in the EBD group was due to complications induced by EBD and failure to achieve sufficient decompression of the future remnant liver. Cholangitis, which is one of the complications that can occur after preoperative biliary drainage, is a notable problem that often requires reintervention. Patients in the EBD group experienced significantly higher rates of cholangitis than those in the PTBD group (PTBD vs EBD: $8.1 \%$ vs $38.6 \%, \mathrm{p}<0.001$ ), consistent with previous reports. ${ }^{27,28}$ This may explain in part why the EBD group had a higher conversion rate than the PTBD group. Indeed, cholangitis is the main complication post-ERCP in patients with perihilar cholangiocarcinoma, especially when contrast medium is injected into a biliary tree that can not subsequently be drained.

Despite the many advantages of PTBD as a preoperative biliary drainage method, it has one great weakness; the possibility of cancer dissemination along the catheter tract. This is an irrevocable event unlike other complications such as cholangitis, hemorrhage, and pancreatitis. Hwang et al. ${ }^{29}$ reported results of PTBD in 231 patients with surgical resected perihilar cholangiocarcinoma; four patients $(1.7 \%)$ experienced cancer recurrence in the PTBD tract a median of 13.5 months after surgery. Takahashi et al. ${ }^{11}$ documented recurrence in the PTBD catheter tract in 23 of 445 patients (5.2\%) with perihilar and distal cholangiocarcinoma who underwent resection following PTBD. The authors suggested that the incidence of PTBD tract recurrence may be underestimated because while catheter-related metastatic deposits can develop at any site, early detection of recurrence at the site where a catheter enters into the biliary tract, such as the skin, abdominal wall, and liver parenchyma, is difficult. In our study, of 52 patients who underwent curative resection in the PTBD group, two (3.8\%) developed catheter tract implantation metastasis during the follow-up period. They showed a relatively short mean time to recurrence compared to the other 28 patients who experienced tumor recurrence in the PTBD group without catheter tract metastasis (10.5 months vs 16.0 months). Previous investigators have speculated that multiple catheter drains and a long duration PTBD procedure are potential risk factors for tract recurrence. ${ }^{11}$ Having to place a catheter more than once is also associated with increased chances of bile spillage, which could induce cancer dissemination. Even though the rate of procedure-related complications such as cholangitis and pancreatitis was relatively high in patients with EBD, our results indicate that complications from EBD were associated with an acceptable morbidity and could be adequately managed. Our study involved nine ERBD and 35 ENBD cases. Among these EBD methods, ERBD has been selected less frequently of late due to a tendency for ERBD to trigger ascending cholangitis caused by retrograde flow of duodenal fluid into the biliary tree. ERBD can also trigger acute obstructive cholangitis more frequently than other EBD methods, because ERBD does not allow for regular irrigation, which prevents obstruction. ENBD is preferable to ERBD in that it has little effect on retrograde cholangitis and postdrainage cholangiography is possible via the nasobiliary tube, albeit with the discomfort of nasal intubation. In the present study, although we found that ERBD was associated with a higher rate of procedure-related cholangitis than ENBD (44.4\% vs $34.3 \%)$, the difference was not statistically significant ( $p=0.702)$. ENBD has gained wide acceptance due to reduced cholangitis compared to ERBD and its lower invasiveness, allowing transhepatic puncture to be avoided and providing an opportunity for contrast cholangiography.

The main limitations of the present study are its retrospective nature and the fact that initial preoperative biliary drainage was conducted in a relatively low proportion of tertiary referral hospitals; there may therefore be selection bias for patients who were preferred for EBD or PTBD. Moreover, the lower rate of initial successful drainage than that reported in previous studies may be due to the lower frequency of tertiary referral centers that made the first drainage attempt in our study than previous studies. Experience conferred by a greater caseload and more experienced endoscopists and radiologists would likely improve the rate of successful biliary decompression on initial drainage. In addition, there could have been operable cases where biliary decompression was attended to, but, for various reasons, unknown to this study, the subsequent surgery was never performed, and due to the retrospective study design, we were unable to address the presence of these situation in our analysis. We recognize that prospective studies are needed to quantitatively compare the two preoperative drainage methods. The present study is significant, however, because it is one of very few studies to investigate clinical outcomes of PTBD and ENBD for preoperative biliary drainage in perihilar cholangiocarcinoma in terms of therapeutic efficacy, safety, and longterm prognosis. Another strength of this study is the inclusion of a relatively large number of patients who underwent surgical 
treatment for perihilar cholangiocarcinoma.

In conclusion, we found that EBD was associated with a higher risk of procedure-related complications, such as cholangitis and pancreatitis, than PTBD, and that PTBD was associated with fewer infectious complications than EBD, resulting in a significantly lower frequency of reintervention after PTBD. However, these complications were readily managed without severe morbidity in most cases. Our results indicate that there may be an advantage to using ENBD rather than ERBD for decompression of the future remnant liver during initial preoperative biliary drainage in patients with resectable perihilar cholangiocarcinoma. PTBD should be considered the next best alternative when ENBD is not feasible or insufficient for biliary decompression because of the real, albeit low risk of lethal catheter tract metastasis.

\section{CONFLICTS OF INTEREST}

No potential conflict of interest relevant to this article was reported.

\section{REFERENCES}

1. Blamey SL, Fearon KC, Gilmour WH, Osborne DH, Carter DC. Prediction of risk in biliary surgery. Br J Surg 1983;70:535-538.

2. Dixon JM, Armstrong CP, Duffy SW, Davies GC. Factors affecting morbidity and mortality after surgery for obstructive jaundice: a review of 373 patients. Gut 1983;24:845-852.

3. Shigeta H, Nagino M, Kamiya J, et al. Bacteremia after hepatectomy: an analysis of a single-center, 10-year experience with 407 patients. Langenbecks Arch Surg 2002;387:117-124.

4. Nimura Y. Preoperative biliary drainage before resection for cholangiocarcinoma (Pro). HPB (Oxford) 2008;10:130-133.

5. Nagino M, Hayakawa N, Nimura Y, Dohke M, Kitagawa S. Percutaneous transhepatic biliary drainage in patients with malignant biliary obstruction of the hepatic confluence. Hepatogastroenterology 1992;39:296-300.

6. Maguchi H, Takahashi K, Katanuma A, et al. Preoperative biliary drainage for hilar cholangiocarcinoma. J Hepatobiliary Pancreat Surg 2007;14:441-446.

7. Kawasaki S, Imamura H, Kobayashi A, Noike T, Miwa S, Miyagawa S. Results of surgical resection for patients with hilar bile duct cancer: application of extended hepatectomy after biliary drainage and hemihepatic portal vein embolization. Ann Surg 2003;238:8492.

8. Kawakami H, Kuwatani M, Onodera M, et al. Endoscopic nasobiliary drainage is the most suitable preoperative biliary drainage method in the management of patients with hilar cholangiocarcinoma. J Gastroenterol 2011;46:242-248.

9. van Delden OM, Lameris JS. Percutaneous drainage and stenting for palliation of malignant bile duct obstruction. Eur Radiol 2008;18:448-456.
10. Nimura Y, Kamiya J, Kondo S, et al. Aggressive preoperative management and extended surgery for hilar cholangiocarcinoma: Nagoya experience. J Hepatobiliary Pancreat Surg 2000;7:155162.

11. Takahashi Y, Nagino M, Nishio H, Ebata T, Igami T, Nimura Y. Percutaneous transhepatic biliary drainage catheter tract recurrence in cholangiocarcinoma. Br J Surg 2010;97:1860-1866.

12. Sakata J, Shirai Y, Wakai T, Nomura T, Sakata E, Hatakeyama K. Catheter tract implantation metastases associated with percutaneous biliary drainage for extrahepatic cholangiocarcinoma. World J Gastroenterol 2005;11:7024-7027.

13. Cotton PB, Lehman G, Vennes J, et al. Endoscopic sphincterotomy complications and their management: an attempt at consensus. Gastrointest Endosc 1991;37:383-393.

14. Dindo D, Demartines N, Clavien PA. Classification of surgical complications: a new proposal with evaluation in a cohort of 6336 patients and results of a survey. Ann Surg 2004;240:205-213.

15. Rahbari NN, Garden OJ, Padbury R, et al. Posthepatectomy liver failure: a definition and grading by the International Study Group of Liver Surgery (ISGLS). Surgery 2011;149:713-724.

16. Kennedy TJ, Yopp A, Qin Y, et al. Role of preoperative biliary drainage of liver remnant prior to extended liver resection for hilar cholangiocarcinoma. HPB (Oxford) 2009;11:445-451.

17. Burke EC, Jarnagin WR, Hochwald SN, Pisters PW, Fong Y, Blumgart LH. Hilar cholangiocarcinoma: patterns of spread, the importance of hepatic resection for curative operation, and a presurgical clinical staging system. Ann Surg 1998;228:385-394.

18. Hemming AW, Reed AI, Fujita S, Foley DP, Howard RJ. Surgical management of hilar cholangiocarcinoma. Ann Surg 2005;241: 693-699.

19. Neuhaus P, Jonas S, Bechstein WO, et al. Extended resections for hilar cholangiocarcinoma. Ann Surg 1999;230:808-818.

20. Sano T, Shimada K, Sakamoto Y, Yamamoto J, Yamasaki S, Kosuge T. One hundred two consecutive hepatobiliary resections for perihilar cholangiocarcinoma with zero mortality. Ann Surg 2006; 244:240-247

21. van der Gaag NA, Kloek JJ, de Castro SM, Busch OR, van Gulik TM, Gouma DJ. Preoperative biliary drainage in patients with obstructive jaundice: history and current status. J Gastrointest Surg 2009;13:814-820.

22. Gundry SR, Strodel WE, Knol JA, Eckhauser FE, Thompson NW. Efficacy of preoperative biliary tract decompression in patients with obstructive jaundice. Arch Surg 1984;119:703-708.

23. Liu CL, Fan ST, Lo CM, Tso WK, Lam CM, Wong J. Improved operative and survival outcomes of surgical treatment for hilar cholangiocarcinoma. Br J Surg 2006;93:1488-1494.

24. Kondo S, Hirano S, Ambo Y, et al. Forty consecutive resections of hilar cholangiocarcinoma with no postoperative mortality and no positive ductal margins: results of a prospective study. Ann Surg 2004;240:95-101

25. Arakura N, Takayama M, Ozaki Y, et al. Efficacy of preoperative endoscopic nasobiliary drainage for hilar cholangiocarcinoma. J 
Hepatobiliary Pancreat Surg 2009;16:473-477.

26. Kloek JJ, van der Gaag NA, Aziz Y, et al. Endoscopic and percutaneous preoperative biliary drainage in patients with suspected hilar cholangiocarcinoma. J Gastrointest Surg 2010;14:119-125.

27. Rerknimitr R, Kladcharoen N, Mahachai V, Kullavanijaya P. Result of endoscopic biliary drainage in hilar cholangiocarcinoma. J Clin Gastroenterol 2004;38:518-523.
28. dos Santos JS, Junior WS, Modena JL, Brunaldi JE, Ceneviva R. Effect of preoperative endoscopic decompression on malignant biliary obstruction and postoperative infection. Hepatogastroenterology 2005;52:45-47.

29. Hwang S, Song GW, Ha TY, et al. Reappraisal of percutaneous transhepatic biliary drainage tract recurrence after resection of perihilar bile duct cancer. World J Surg 2012;36:379-385. 